Encontro Nacional de

Economia Industrial e Inovação
Indústria e Desenvolvimento Econômico:

desafios e perspectivas

18 a 20 de setembro de 2018

Uberlândia - Minas Gerais

\title{
O fortalecimento do segmento de base química e biotecnológica do Complexo Industrial da Saúde por meio das Parcerias para o Desenvolvimento Produtivo
}

\section{Alexandra Patricia Albareda ${ }^{1}$ Ricardo Lobato Torres ${ }^{2}$}

\section{Área temática 6.1 - Políticas industriais e Comerciais \\ Classificação JEL L52 - Industrial Policy, Sectoral Planning Methods; O25 - Industrial Policy e H51 - Government Expenditure in Health}

Resumo: O presente artigo busca verificar se as Parcerias para o Desenvolvimento Produtivo (PDP) são um instrumento de fortalecimento do segmento de base química e biotecnológica do Complexo Econômico-Industrial da Saúde (CEIS). Há alguns anos a saúde no Brasil atingiu uma dimensão mais abrangente, sendo reconhecida como um agregado macroeconômico, indutora de crescimento econômico e social. Assim, as políticas industriais apresentadas nos últimos anos demonstraram uma preocupação clara e específica com o setor de saúde. O CEIS é apresentado como um conceito que busca uma junção da área da saúde e do complexo industrial. E, de forma a fortalecer o CEIS, surgem as PDP, com o objetivo de atender as demandas do Sistema Único de Saúde (SUS) ao internalizar o desenvolvimento dos produtos essenciais ao cumprimento da garantia de acesso universal e equitativo à saúde. Verifica-se, por meio de análise bibliográfica, documental e de dados secundários que as PDP têm se mostrado uma ferramenta essencial ao fortalecimento do CEIS e ao desenvolvimento econômico e social do país.

Palavras Chave: Política Industrial; Política de Saúde; Inovação; Indústria Farmacêutica; Sistema Único de Saúde; Desenvolvimento Econômico.

Abstract: This article is intended at verifying if the Productive Development Partnerships (PDP’s) are an instrument to strengthen the Chemistry and Biotechnology segment of the Health's Economic and Industrial Complex (CEIS, in Portuguese). Over the last years healthcare in Brazil has reached a broader dimension, being recognized as a macroeconomic driver, one that can leverage economic and social growth. Hence, policies aimed at the industry sector in the course of the last years have demonstrated a clear and specific attention towards the healthcare segment. CEIS is introduced as a concept that seeks to unite the areas of healthcare and industrial complex. And, as a means to strengthening CEIS, PDP's are brought to life with the objective of responding to the demands by Unique Healthcare System (SUS) by assuming the development of the products that are essential to granting universal and equalitarian access to healthcare. Bibliographic, documental and secondary data's analysis indicates that PDP’s have proved to be an essential tool for the consolidation of CEIS and the economic and social development of the country.

Keywords: Industrial Policy; Health Policy; Innovation; Pharmaceutical Industry; Unique Health System; Economic Development.

\footnotetext{
${ }^{1}$ Mestranda do curso de Pós-Graduação em Planejamento e Governança Pública - UTFPR - alexandra.albareda@gmail.com.

${ }^{2}$ Professor do curso de Pós-Graduação em Planejamento e Governança Pública - UTFPR - rltorres@utfpr.edu.br.
} 


\section{INTRODUÇÃO}

Nos últimos anos, a visão sobre a saúde no Brasil começou a tomar novos rumos. A sua compreensão como a simples ausência de doença tem dado espaço a uma dimensão mais abrangente, e sua importância estratégica vem sendo reconhecida em diversos estudos e campos de atuação.

Reconhece-se o papel fundamental desempenhado pela saúde no desenvolvimento de uma nação. Dessa forma, o setor da saúde tem passado por profundas transformações estruturais ao ser invadido pela lógica empresarial capitalista, que acaba por incorporá-lo à dinâmica econômica com base na premissa de que "a saúde desempenha importante papel como indutora de crescimento econômico e competitividade nacional” (GADELHA, 2010).

Nesse sentido, Gadelha (2003) apresenta o conceito de Complexo Econômico Industrial da Saúde (CEIS), que representa um olhar diferenciado na forma de abordar esse setor, "representando uma percepção da área como um conjunto interligado de produção de bens e serviços em saúde que se movem no contexto da dinâmica capitalista” (GADELHA, 2003).

O CEIS engloba em seu conjunto atividades industriais - de base química e biotecnológica e de equipamentos médico-hospitalares - e de serviços que, segundo Costa, Metten e Delgado (2016), são responsáveis atualmente por cerca de $9 \%$ do Produto Interno Bruto (PIB) nacional, de 10\% dos empregos qualificados e de mais de $25 \%$ do investimento em pesquisa e desenvolvimento (P\&D) no país. O segmento de base química e biotecnológica é responsável pela produção de medicamentos, fármacos, vacinas e soros, hemoderivados e reagentes para diagnóstico.

Gadelha já apontava em 2010 a necessidade do incentivo à nacionalização desse segmento industrial, em virtude dos altos custos decorrentes da importação. Dados apurados pela Associação Brasileira das Indústrias de Química Fina, Biotecnologia e suas Especialidades (ABIFINA) mostram que, em 2017, o país importou US\$ 3.343.222,00 em medicamentos, enquanto que o valor da exportação se resumiu a singelos US\$ 906.476,00, tendo o saldo comercial fechado o ano em US\$ -2.436.746,00.

É apenas a partir dos anos 2000 que se verifica o surgimento de políticas públicas de apoio ao CEIS baseadas na articulação de uma rede institucional intersetorial e, como resultado,

“observou-se uma série de estratégias de fortalecimento da base produtiva da saúde, destacando-se a intensificação do uso do poder de compra do Estado e a transferência de tecnologia para os laboratórios nacionais, materializadas majoritariamente nas Parcerias para o Desenvolvimento Produtivo (PDP)" (COSTA, METTEN e DELGADO, 2016).

As Parcerias para o Desenvolvimento Produtivo (PDP) foram regulamentadas, inicialmente, por meio da Portaria $\mathrm{n}^{\circ}$ 837, de 18 de abril de 2012, que estabeleceu as PDP como

parcerias realizadas entre instituições públicas e entidades privadas com vistas ao acesso a tecnologias prioritárias, à redução da vulnerabilidade do Sistema Único de Saúde (SUS) a longo prazo e à racionalização e redução de preços de produtos estratégicos para saúde, com o comprometimento de internalizar e desenvolver novas tecnologias estratégicas e de valor agregado elevado (BRASIL, 2012).

Essa Portaria inclui, entre os produtos e bens a serem produzidos por meio das parcerias, os fármacos e medicamentos. Como objetivos elencados, destaca-se o foco a diminuição dos custos para o SUS por meio da viabilização da produção nacional, o que acarreta também na diminuição da vulnerabilidade do SUS ao conquistar o pleno abastecimento das suas necessidades.

Em 2014 foi publicada a Portaria ${ }^{\circ}$ 2.531, de 12 de novembro, que redefiniu o estabelecimento das PDP e cujo conceito passou a ser, conforme o inciso I do art. $2^{\circ}$ :

(...) parcerias que envolvem a cooperação mediante acordo entre instituições públicas e entre instituições públicas e entidades privadas para desenvolvimento, 
transferência e absorção de tecnologia, produção, capacitação produtiva e tecnológica do País em produtos estratégicos para atendimento às demandas do SUS (BRASIL, 2014).

Essa Portaria acabou sendo englobada pela Portaria de Consolidação GM/MS n ${ }^{0}$ 5/2017, que consolida as normas sobre as ações e os serviços de saúde do SUS. Assim, a Portaria $\mathrm{n}^{0} 2.531$ passou a compor o Anexo XCV da Portaria de Consolidação, não havendo mudanças em seu conteúdo (BRASIL, 2017).

Conforme aponta Torres (2016),

as PDPs (...) visam capacitar os laboratórios farmacêuticos públicos e privados nacionais a produzir medicamentos de maior complexidade tecnológica, através de acordos de transferência de tecnologia junto aos laboratórios multinacionais. Grosso modo, trata-se de uma política que utiliza o poder de compra do Estado para criar um estímulo ao investimento por parte dos laboratórios nacionais ao mesmo tempo em que "reparte" o mercado público entre três atores: o laboratório público da rede oficial (chamados de laboratórios farmacêuticos oficiais - LFO), o laboratório privado nacional (leia-se empresas cujo controle do capital é brasileiro - LPN) e o laboratório farmacêutico multinacional (LFM) (TORRES, 2016).

Dessa forma, este trabalho busca verificar, por meio de análise bibliográfica e documental e análise de dados secundários que subsidiam o tema abordado, se é possível denotar e avaliar um fortalecimento do segmento de base química e biotecnológica do CEIS por meio das PDP firmadas desde seu surgimento.

De maneira a cumprir com o objetivo proposto, o trabalho se divide em sete seções: após esta breve introdução, a segunda seção aborda um breve histórico das políticas industriais voltadas ao setor de saúde no Brasil. A terceira traça um breve paralelo entre saúde e desenvolvimento nacional. A quarta apresenta um referencial teórico sobre o CEIS (especificamente em relação ao segmento de base química e biotecnológica). A quinta seção apresenta as PDP, seu funcionamento e demanda do SUS. A sexta seção mostra as análises realizadas por meio dos dados obtidos na pesquisa e a sétima e última seção traz as considerações finais, na qual são apresentadas as conclusões sobre a pesquisa realizada.

\section{POLÍTICAS INDUSTRIAIS NA ÁREA DA SAÚDE}

Antes de buscar elencar as políticas industriais na área da saúde que foram implementadas no Brasil, é necessário compreender o que são políticas industriais (PI) e qual a visão dos economistas a seu respeito.

Ao falar sobre PI no Brasil, há ainda uma visão restrita que remonta ao início da industrialização no país, quando o foco das políticas industriais era o desenvolvimento da infraestrutura nacional e a política de substituição de importações (SI), o que, de certa forma, serviu para atrair o investimento estrangeiro em virtude do atrativo exercido pela grande demanda interna e da concessão de isenções e subsídios aos investidores. Isso acarretou na criação de uma base industrial diversificada, porém, o foco excessivo no cliente interno tornou as indústrias pouco competitivas internacionalmente e demasiadamente voltadas à produção manufatureira.

Essa visão começou a mudar a partir dos anos 2000, por meio da adoção de políticas industriais que trouxeram à tona a preocupação com a inovação e a competitividade da indústria a nível internacional, ao mesmo tempo em que surge a preocupação com o desenvolvimento de uma base produtiva nacional que possibilite o atendimento às necessidades locais e a fuga da política de SI.

Esse foco demonstra uma adesão às mudanças estratégicas promovidas pela Comissão Econômica para a América Latina e o Caribe (CEPAL), que a partir dos anos 90 adota um viés mais schumpeteriano aliado ao disseminado estruturalismo da Comissão, o que culminou em uma fórmula neoestruturalista 
que, baseada nas contribuições de Fajnzylber, voltou sua preocupação à transformação produtiva com equidade, por meio, principalmente, da inovação (BIELSCHOWSKY, 2009).

Conforme explica Perez (2009), "Schumpeter is among the few modern economists to put technical change and entrepreneurship at the root of economic growth". O foco desse economista era explicar o papel da inovação no crescimento econômico, sendo que os neoschumpeterianos aprofundaram seus estudos ao pesquisar a evolução tecnológica, identificando processos de inovação ao longo da história.

Assim, as políticas industriais visam, especialmente, impulsionar o desenvolvimento de um país. Segundo Ferraz, Paula e Kupfer (in KUPFER e HASENCLEVER, 2002), do ponto de vista conceitual, política industrial deve ser entendida como

o conjunto de incentivos e regulações associadas a ações públicas, que podem afetar a alocação inter e intraindustrial de recursos, influenciando a estrutura produtiva e patrimonial, a conduta e o desempenho dos agentes econômicos em um determinado espaço nacional (FERRAZ, PAULA e KUPFER in KUPFER e HASENCLEVER, 2002).

Ainda não há um consenso a respeito de como deve se dar sua formulação e implementação, pois, conforme sugerem Suzigan e Furtado (2006), há duas principais visões sobre seus fundamentos teóricos. Por um lado, um grupo mais liberal sugere que as políticas industriais devem ser formuladas para a correção de falhas de mercado, o que torna a política horizontal, ou seja, não restrita a setores específicos, e cuja implementação se dá apenas na verificação de imperfeições de mercado. Por outro lado, os neoschumpeterianos baseiam seus fundamentos em criteriosa análise dos fenômenos econômicos, argumentando que a formulação de PI deve se voltar a setores estratégicos ao crescimento e desenvolvimento nacional.

Considerando o escopo deste artigo, adotar-se-á a visão neoschumpeteriana de PI, pois ao delimitar a análise ao setor da saúde e, especificamente, ao CEIS, será verificada a importância das políticas industriais no fortalecimento desse segmento, mostrando que a análise criteriosa dos aspectos econômicos é essencial para a formulação de políticas voltadas à inovação, que apresentem objetivos concisos e necessários ao desenvolvimento.

Ao mesmo tempo, defende-se a ideia de um desenvolvimento capitalista nacionalista em detrimento ao desenvolvimento dependente que vigora no Brasil, que leva o país a enfrentar uma crescente desigualdade social e o torna dependente dos investimentos estrangeiros para obter crescimento econômico (KOHLI, 2012)

A predominância neoschumpeteriana fica clara já na adoção da que se tornou a primeira política industrial nacional com foco central na inovação: a Política Industrial, Tecnológica e de Comércio Exterior (PITCE), formulada em novembro de 2003 e anunciada em março de 2004.

A PITCE apresentou uma série de ações que deveriam ser concentradas em determinadas áreas, apresentando a indústria de fármacos e medicamentos como uma das "atividades importantes para o futuro do país e [com] potencial para o desenvolvimento de vantagens comparativas dinâmicas“ (BRASIL, 2003).

Conforme balanço publicado em 2006 pela Agência Brasileira de Desenvolvimento Industrial (ABDI), os medicamentos fazem parte dos bens sociais e estratégicos. No entanto, o setor sofreu forte desindustrialização nos anos 90, o que tornou essa indústria vulnerável e dependente do mercado internacional. Para reverter essa situação, um dos pilares propostos pela PITCE foi estimular a produção nacional de fármacos e medicamentos, por meio da criação, pelo BNDES, de uma linha especial de crédito: o PROFARMA - Programa de Apoio ao Desenvolvimento da Cadeia Produtiva Farmacêutica.

Logo após a PITCE, ainda em 2004, foi lançada a Política Nacional de Ciência, Tecnologia e Inovação em Saúde (PNCTIS), sendo parte integrante da Política Nacional de Saúde, formulada no âmbito do Sistema Único de Saúde (SUS), tendo como referência seus três princípios: universalidade, integralidade e equidade. Varrichio (2017) aponta que já na PNCTIS adotou-se o conceito de complexo produtivo em saúde, composto pelos seguintes grupos: pela indústria química, farmacêutica e de 
biotecnologia; pela indústria mecânica, eletrônica e de materiais; e pelos prestadores de serviço, sendo que mais tarde houve ampliação da nomenclatura para Complexo Econômico-Industrial da Saúde (CEIS).

O maior objetivo da PNCTIS é "contribuir para que o desenvolvimento nacional se faça de modo sustentável, e com apoio na produção de conhecimentos técnicos e científicos ajustados às necessidades econômicas, sociais, culturais e políticas do País” (BRASIL, 2008). Para tanto, a política confere especial importância à apropriação de conhecimentos e tecnologias que reduzam as desigualdades sociais em saúde.

Segundo Torres (2016), “em 2008, com a revisão da política industrial do governo federal, foi lançada a Política de Desenvolvimento Produtivo (PDP), que ampliou o leque de setores considerados estratégicos”. Sua vigência foi até 2010, e seu principal objetivo era dar sustentabilidade ao crescimento econômico, aumentar os investimentos produtivos e as taxas de crescimento econômico. A exemplo da PITCE, a PDP também englobou um conjunto de medidas que visavam o fortalecimento do país por meio da indústria. A diferença nessa política foi trazer o Complexo Industrial da Saúde (CIS) como uma das áreas estratégicas às quais deveriam ser direcionados os esforços, incluindo, além da produção de fármacos e medicamentos, a de equipamentos médico-hospitalares.

Com esse objetivo em vista, o BNDES renova, em 2007, o PROFARMA, que passa então a se chamar Programa de Apoio ao Desenvolvimento do Complexo Industrial da Saúde, contando com um orçamento de R\$ 3 bi, 50\% dos quais seria destinado exclusivamente para financiamento à inovação (G1, 2007).

Em 2008 é publicada, em consonância com a política industrial vigente, a Portaria $n^{0}$ 374, de 287 de fevereiro, que Institui no âmbito do Sistema Único de Saúde - SUS, o Programa Nacional de Fomento à Produção Pública e Inovação no Complexo Industrial da Saúde. De acordo ao art. $2^{\circ}$ da portaria, o objetivo do programa é

promover o fortalecimento e a modernização do conjunto de laboratórios públicos encarregados da produção de medicamentos e imunobiológicos de relevância estratégica para o Sistema Único de Saúde, por intermédio da ampliação da participação no Complexo Produtivo da Saúde, do aumento da capacidade inovadora e da mudança de patamar competitivo, contribuindo para a redução da defasagem tecnológica existente e o desenvolvimento econômico, científico e tecnológico do País (BRASIL, 2008a).

No mesmo ano, publica-se o Decreto DNN, de 12 de maio, o qual cria, no âmbito do Ministério da Saúde, o Grupo Executivo do Complexo Industrial de Saúde (GECIS), destinado a

promover medidas e ações concretas visando à criação e implementação do marco regulatório brasileiro referente à estratégia de desenvolvimento do Governo Federal para a área da saúde, segundo as diretrizes das políticas nacionais de fortalecimento do complexo produtivo e de inovação em saúde (BRASIL, 2008b).

Esse Decreto foi revogado em 2017, tendo o GECIS sua regulamentação atualizada por meio do Decreto $\mathrm{n}^{\circ} 9.245 / 2017$, passando a ser objetivos do Grupo:

I - promover a articulação dos órgãos e das entidades públicos, da indústria química, farmacêutica, de biotecnologia, mecânica, eletrônica e de materiais para a saúde e das entidades que atuem em pesquisa, inovação, desenvolvimento, produção e prestação de serviços na área da saúde, no âmbito da PNITS;

II - fomentar ambiente propício ao desenvolvimento industrial e tecnológico no CIS para a ampliação do acesso a produtos e serviços estratégicos para o SUS; e III - promover ambiente de segurança jurídica e institucional que favoreça o investimento produtivo e em pesquisa, inovação e desenvolvimento no País na área da saúde (BRASIL, 2017). 
O terceiro programa governamental que mostrou preocupação com o setor da saúde foi o Plano Brasil Maior (PBM), editado em 2011 com vigência até 2014, com os objetivos de “acelerar o crescimento do investimento produtivo e o esforço tecnológico e de inovação das empresas nacionais, e aumentar a competitividade dos bens e serviços nacionais” (BRASIL, 2011).

De forma a auxiliar na formulação de programas e projetos a partir de diretrizes setoriais, temáticos e que englobem mais de um setor, o PBM dividiu os setores estratégicos em cinco blocos, sendo o primeiro deles o de sistemas da mecânica, eletroeletrônica e saúde, dentre os quais é citado o Complexo Industrial da Saúde. Torres (2016) afirma que o reforço das políticas industriais direcionadas ao CIS trazido pelo PBM

(...) se traduziu no lançamento do Programa Inova Saúde da Financiadora de Estudos e Projetos (FINEP), cujo foco foi a provisão de subvenção econômica para empresas públicas e privadas no país com propostas de projetos de inovação tecnológica em áreas relacionadas à saúde, com destaque para biofármacos, farmoquímicos e medicamentos. Destaca-se, ainda, que em 2013 o BNDES lançou a terceira fase do PROFARMA, visando o financiamento de projetos empresariais de biotecnologia farmacêutica e biomédica (TORRES, 2016, GRIFO NOSSO).

Foi durante a vigência do PBM que foram regulamentadas as Parcerias para o Desenvolvimento Produtivo (PDP), que são apontadas por alguns autores como uma das melhores estratégias no intuito de ampliar o acesso a medicamentos e produtos para a saúde considerados estratégicos para o SUS.

Assim, em 2012, publica-se a Portaria $\mathrm{n}^{0}$ 506, de 21 de março, a qual institui o Programa para o Desenvolvimento do Complexo Industrial da Saúde (PROCIS) e seu Comitê Gestor, com o objetivo geral de "fortalecer os produtores públicos e a infraestrutura de produção e inovação em saúde do setor público” (BRASIL, 2012).

São traçados, ainda, sete objetivos específicos em seu art. $2^{\circ}$, entre os quais se destacam, respeitando o escopo deste trabalho:

III - fortalecer as parcerias para o desenvolvimento produtivo visando desenvolver e absorver produtos estratégicos para o Sistema Único de Saúde;

V - apoiar o desenvolvimento tecnológico e a transferência de tecnologias, estratégicos para o Sistema Único de Saúde (SUS);

VII - apoiar a infraestrutura pública de tecnologia e inovação para suporte à produção no país de produtos estratégicos para o SUS (BRASIL, 2012, GRIFO NOSSO).

Denota-se, por meio de tudo o que foi exposto, um forte intento governamental em resgatar as políticas industriais como uma das estratégias propulsoras do desenvolvimento nacional. Verifica-se também a importância atribuída ao setor da saúde nas últimas políticas, o que pode ser compreendido como uma nova visão da saúde dentro das perspectivas de crescimento e desenvolvimento econômico de uma nação.

\section{SAÚDE E DESENVOLVIMENTO NACIONAL}

Gadelha (2007) situa a saúde como uma “condição de cidadania, sendo parte inerente do próprio conceito do desenvolvimento. Não há país que possa ser considerado como desenvolvido com a saúde precária”.

Carrara e Ventura (2012), afirmam que "saúde e desenvolvimento estão intimamente ligados, uma vez que o processo de desenvolvimento envolve a consolidação do direito à saúde”. 
Dória et al. (in Hasenclever et al., 2016), reconhecem a saúde como um agregado macroeconômico, enfatizando que "a importância da produção da saúde transcende o bem-estar social". Segundo os autores, "os bens e serviços da saúde têm alta representatividade no produto, no emprego, no consumo e na balança comercial dos países”, o que torna essencial a análise dessas variáveis na formulação de políticas públicas.

Ainda, conforme relatório publicado pela Organização Mundial de Saúde (OMS), intitulado Saúde e Macroeconomia: Investindo em Saúde para o Desenvolvimento Econômico, em termos econômicos, a saúde, junto com a educação, constituem os dois pilares do capital humano. Afirma-se, inclusive, que "disease reduces annual incomes of society, the lifetime incomes of individuals, and prospects for economic growth” (OMS, 2001).

O relatório vai mais além ao garantir que "because disease weighs so heavily on economic development, investing in health is an important component of an overall development strategy" (OMS, 2001), isso porque, para a OMS, a situação da saúde em uma nação parece explicar uma parte importante da diferença nas taxas de desenvolvimento econômico.

Ao reconhecer a saúde como um dos fatores determinantes para alavancar o crescimento e desenvolvimento econômico de uma região, há de se admitir a importância da formulação e implementação das políticas de saúde.

Uma das maiores conquistas sociais na histórica política do Brasil é, sem dúvida, a promulgação da Constituição Federal de 1988 (CF/88), batizada como “Constituição cidadã”, por priorizar, em seu texto, diversos direitos sociais, o que, conforme aponta Castro (in GIAMBIAGI, 2011), "se manifesta, inclusive, na própria ordenação dos capítulos do Novo Texto" ao trazer já no seu segundo título os Direitos e Garantias Individuais, sendo a saúde um deles.

Dessa maneira, a saúde se torna, conforme o art. 196 da CF/88 "direito de todos e dever do Estado, garantido mediante políticas sociais e econômicas que visem à redução do risco de doença e de outros agravos e ao acesso universal e igualitário às ações e serviços para sua promoção, proteção e recuperação” (BRASIL, 1988).

Cria-se, assim, o Sistema Único de Saúde (SUS), com financiamento proveniente do orçamento da seguridade social, da União, dos Estados, do Distrito Federal e dos Municípios, além de outras fontes, e com oito atribuições definidas já na Carta Magna, das quais se destacam especificamente para o objeto deste artigo:

I - controlar e fiscalizar procedimentos, produtos e substâncias de interesse para a saúde e participar da produção de medicamentos, equipamentos, imunobiológicos, hemoderivados e outros insumos;

V - incrementar, em sua área de atuação, o desenvolvimento científico e tecnológico e a inovação; (Redação dada pela EC n. 85/2015) (BRASIL, 1988, GRIFO NOSSO).

De forma a regulamentar o funcionamento do SUS foi editada a Lei ${ }^{\circ}$ 8.080, de 19 de setembro de 1990, que “dispõe sobre as condições para promoção, proteção e recuperação da saúde, a organização e o funcionamento dos serviços correspondentes e dá outras providências”.

Essa lei traz, em seu capítulo I, as atribuições concernentes ao SUS, entre as quais são destacadas, novamente no intuito de fazer jus ao tema proposto neste trabalho, a execução de ações de assistência terapêutica integral, inclusive farmacêutica, e a formulação de política de medicamentos, além da participação em sua produção (BRASIL, 1990).

Sendo assim, em 2001 a assistência farmacêutica foi garantida por meio da Política Nacional de Medicamentos, com o propósito de "garantir a necessária segurança, eficácia e qualidade dos medicamentos, a promoção do uso racional e o acesso da população àqueles considerados essenciais" (BRASIL, 2001), apresentando entre suas diretrizes a necessidade do desenvolvimento científico e tecnológico e da promoção da produção de medicamentos no país.

Neste ponto, destaca-se uma frase específica, constante no art. $3^{\circ}$ da lei ${ }^{\circ} 8.080$, que condiz com o exposto neste apartado: “Os níveis de saúde expressam a organização social e econômica do País”. Pode- 
se inferir que essa afirmação demonstra, per se, a importância atribuída à saúde no desenvolvimento econômico da nação.

Não é de se estranhar, portanto, que o tema saúde tenha começado a ser reconhecido e estudado em outros campos de atuação, sendo um deles a economia. Dessa forma, ela começou a ser compreendida como necessária ao crescimento e desenvolvimento nacional.

Conforme verificado tanto na Carta Magna, como na lei que regulamentou o SUS, a assistência farmacêutica, a produção de medicamentos e a inovação são destacados como parte da política social que garante uma saúde universal. Sendo assim, as políticas industriais direcionadas ao setor da saúde, em particular ao segmento da base química e biotecnológica, cumprem um papel determinante na diminuição da vulnerabilidade do SUS e busca pelo cumprimento da garantia constitucional à saúde.

Em uma análise econômica, a promoção de políticas industriais voltadas ao segmento da base química e biotecnológica constitui uma ferramenta para o abandono do subdesenvolvimento ao buscar aliar a saúde ao desenvolvimento tecnológico e à inovação, tentando criar uma indústria nacional forte e independente, tendo em vista que a dependência do país em relação às nações desenvolvidas acarreta em aumento de custos com importações de produtos que poderiam ser fabricados nacionalmente e em uma estagnação estrutural ao desestimular a evolução e a inovação.

\section{O COMPLEXO ECONÔMICO INDUSTRIAL DA SAÚDE}

Ao compreender o setor de saúde como parte inerente do desenvolvimento econômico do país, há uma necessidade de repensar a estrutura da economia política de forma a aliar esses dois conceitos em prol do desenvolvimento social.

Para Gadelha (2012), “a saúde como qualidade de vida implica pensar em sua conexão estrutural com o desenvolvimento econômico, a equidade, a sustentabilidade ambiental e a mobilização política da sociedade". Para o autor, no âmbito da economia política, há uma necessidade de recuperação do pensamento desenvolvimentista, de pensar em aprendizado e inovação para garantia do desenvolvimento.

É nessa dimensão que se coloca o tema do Complexo Econômico-industrial da Saúde (CEIS) (...). Em substância, aponta a necessidade de uma mudança profunda na estrutura econômica brasileira que permita, mediante um intenso processo de inovação, adensar o tecido produtivo e direcioná-lo para compatibilizar a estrutura de oferta com a demanda social de saúde (GADELHA, 2012).

Buscando uma definição de Complexo Industrial da Saúde (CIS ou CEIS) ${ }^{3}$, verifica-se que Gadelha (2003) introduz esse conceito ao buscar uma junção da área da saúde e do complexo industrial, mostrando que não há como pensar em desenvolvimento nacional sem articular as políticas industriais e tecnológicas com a política de saúde, reconhecendo dessa forma, inclusive, a natureza capitalista da área da saúde. Conforme afirma o autor:

Reconhecer a natureza capitalista da área da saúde, a produção em massa, a lógica empresarial e financeira e, sobretudo, a dinâmica das inovações é essencial para a concepção de políticas que almejem atenuar o descolamento inerente do sistema capitalista entre a busca do lucro e de mercados e o atendimento às necessidades sociais e individuais. Não é desconsiderando ou negando a dinâmica capitalista que se poderá conceber políticas sociais adequadas. Ao contrário, somente pela compreensão de sua lógica de funcionamento é possível buscar meios eficazes

3 A conceituação do Complexo Industrial da Saúde (CIS) ou Complexo Produtivo da Saúde, proposta por Gadelha, 2002 e 2003, é rigorosamente idêntica à do conceito de Complexo Econômico-Industrial da Saúde (CEIS). Esta nova terminologia é proposta em decorrência do fato de que parte da aplicação do termo "CIS" acabou restringindo-se aos segmentos industriais, atenuando o caráter sistêmico da abordagem que incorpora também o segmento de serviços em saúde (GADELHA, 2010). 
para que finalidades sociais sejam atingidas nos marcos deste sistema (GADELHA, 2003).

Assim sendo,

O CEIS representa a oportunidade de superação da tensão observada entre a lógica econômica e a sanitária no que se refere a políticas de desenvolvimento para a saúde, dado o caráter complexo, porém sistêmico, que relaciona estas lógicas sociais e produtivas. Na realidade, a construção de um sistema universal de saúde, que atenda à demanda sanitária da população, pressupõe a expansão da base produtiva da saúde. A histórica inobservância desta relação levou a que o desenvolvimento deste complexo produtivo ocorresse sem se considerar seu caráter sistêmico, prejudicando a capacidade de entrega de bens e serviços de saúde efetivos e impedindo a observação de um desenvolvimento produtivo da base do CEIS com satisfatório resultado social e econômico (GADELHA, 2010).

A concepção do CEIS, aliada à percepção da saúde como fator chave para o crescimento e desenvolvimento nacional, torna-se extremamente relevante em um país no qual a saúde tornou-se, a partir da sua última Constituição, publicada em 1988, um direito de todos e dever do Estado e, que para esse fim, criou o Sistema Único de Saúde (SUS), pois seu potencial econômico e social pode auxiliar a reduzir a vulnerabilidade do SUS a cumprir seus princípios.

Conforme disposto na seção introdutória deste trabalho, o CEIS é formado por segmentos industriais de base química e biotecnológica (indústria farmacêutica, vacinas, hemoderivados e reagentes para diagnóstico), de base mecânica, eletrônica e de materiais (equipamentos e materiais médicos) e pelo segmento de serviços, sendo que, neste artigo, o enfoque se direciona ao segmento de base química e biotecnológica, em especial à indústria farmacêutica.

\section{AS PARCERIAS PARA O DESENVOLVIMENTO PRODUTIVO}

Conforme explicado anteriormente, as Parcerias para o Desenvolvimento Produtivo (PDP) foram regulamentadas, inicialmente, por meio da Portaria $\mathrm{n}^{\circ}$ 837, de 18 de abril de 2012, redefinidas por meio da Portaria $\mathrm{n}^{\circ}$ 2.531, de 12 de novembro e finalmente incorporadas ao Anexo XCV da Portaria de Consolidação GM/MS n ${ }^{\circ}$ 5/2017. Sua implementação "visa ampliar o acesso a medicamentos e produtos para saúde considerados estratégicos para o Sistema Único de Saúde (SUS), por meio do fortalecimento do complexo industrial do país” (BRASIL, 2018).

Segundo Varrichio (2017),

as PDP são um mecanismo de política industrial utilizado na saúde, que envolve uma série de contrapartidas para que sejam realizadas as compras públicas de fármacos, medicamentos e equipamentos para o SUS. Desta forma, garante-se a internalização da produção e a transferência de tecnologia para um produtor nacional durante o contrato de parceria em que são concretizadas as compras governamentais do SUS (VARRICHIO, 2017).

Sendo a assistência farmacêutica, a produção de medicamentos e a inovação destacados como parte da política social que garante uma saúde universal, é necessário que sejam elaboradas políticas que permitam atingir essa finalidade.

Assim, as PDPs constituem, em sua essência, um instrumento que propicia uma política desenvolvimentista de saúde, inserida em uma política industrial "voltada para setores específicos e para empresas com capacidade de competição internacional” (BRESSER-PEREIRA, 2010).

O Plano Nacional de Saúde 2012-2015 (PNS) apresenta como objetivo o aperfeiçoamento do SUS de forma a contribuir para a melhoria das condições de saúde da população, assim como da qualidade de 
vida e redução das desigualdades. Para isso, foram estabelecidas catorze diretrizes, entre as quais se destacam duas, a de número oito: Garantia da assistência farmacêutica no âmbito do SUS; e a número dez: Fortalecimento do complexo produtivo e de ciência, tecnologia e inovação em saúde como vetor estruturante da agenda nacional de desenvolvimento econômico, social e sustentável, com redução da vulnerabilidade do acesso à saúde (BRASIL, 2011).

Nesse contexto, reconhece-se que, apesar da necessidade constitucional de garantir um acesso gratuito e universal à saúde, assim como assegurar o acesso aos medicamentos essenciais, o país carece de infraestrutura, conhecimento e tecnologia suficiente para fazer esse papel sem tornar-se dependente do mercado internacional, mostrando a importância de pensar em políticas que diminuam a vulnerabilidade do SUS.

Ao falar especificamente do segmento de base química e biotecnológica, evidencia-se a relevância das políticas industriais aliadas às PDP. Ao verificar os números referentes às importações e exportações de medicamentos, farmoquímicos e vacinas humanas, a ABIFINA apresenta estatísticas detalhadas desde o ano de 2010, cujos números são apresentados na tabela 1 abaixo:

Tabela 1 - Importações e exportações de medicamentos, farmoquímicos e vacinas humanas (20102017) - valores em US\$ MIL

\begin{tabular}{|c|c|c|c|c|c|}
\hline & & Farmoquímicos & Medicamentos & $\begin{array}{l}\text { Vacinas } \\
\text { Humanas }\end{array}$ & TOTAL \\
\hline \multirow{2}{*}{2010} & Importação & 2.362 .200 & 3.244 .982 & 1.097.300 & 6.704 .482 \\
\hline & Exportação & 514.800 & 910.629 & 23.037 & 1.148.466 \\
\hline \multirow{2}{*}{2011} & Importação & 2.483 .700 & 3.597 .675 & 559.539 & 6.640 .914 \\
\hline & Exportação & 807.700 & 1.061 .566 & 26.238 & 1.895 .504 \\
\hline \multirow{2}{*}{2012} & Importação & 2.535 .200 & 3.681 .484 & 584.883 & 6.801.567 \\
\hline & Exportação & 757.300 & 1.114 .552 & 20.539 & 1.892 .391 \\
\hline \multirow{2}{*}{2013} & Importação & 2.791 .400 & 3.833 .684 & 672.553 & 7.297.637 \\
\hline & Exportação & 642.600 & 1.144 .264 & 18.909 & 1.805 .773 \\
\hline \multirow{2}{*}{2014} & Importação & 2.716 .300 & 3.797 .252 & 891.929 & 7.405 .481 \\
\hline & Exportação & 561.400 & 1.216 .136 & 11.849 & 1.789 .385 \\
\hline \multirow{2}{*}{2015} & Importação & 2.410 .200 & 3.377 .641 & 620.615 & 6.408 .456 \\
\hline & Exportação & 515.600 & 985.544 & 15.458 & 1.516 .602 \\
\hline \multirow{2}{*}{2016} & Importação & 2.388 .600 & 3.411 .030 & 700.298 & 6.499 .928 \\
\hline & Exportação & 546.000 & 885.763 & 6.895 & 1.438 .568 \\
\hline \multirow{2}{*}{2017} & Importação & 2.594 .100 & 3.343 .222 & 661.717 & 6.599 .039 \\
\hline & Exportação & 610.200 & 906.476 & 22.608 & 1.539 .284 \\
\hline
\end{tabular}

Fonte: Elaborada pelos autores com base nos dados extraídos da ABIFINA.

A título de ilustração, são apresentados abaixo três gráficos (um para cada setor do segmento), de forma a mostrar de uma maneira mais perceptível como são acentuadas as diferenças expostas na tabela 1. 


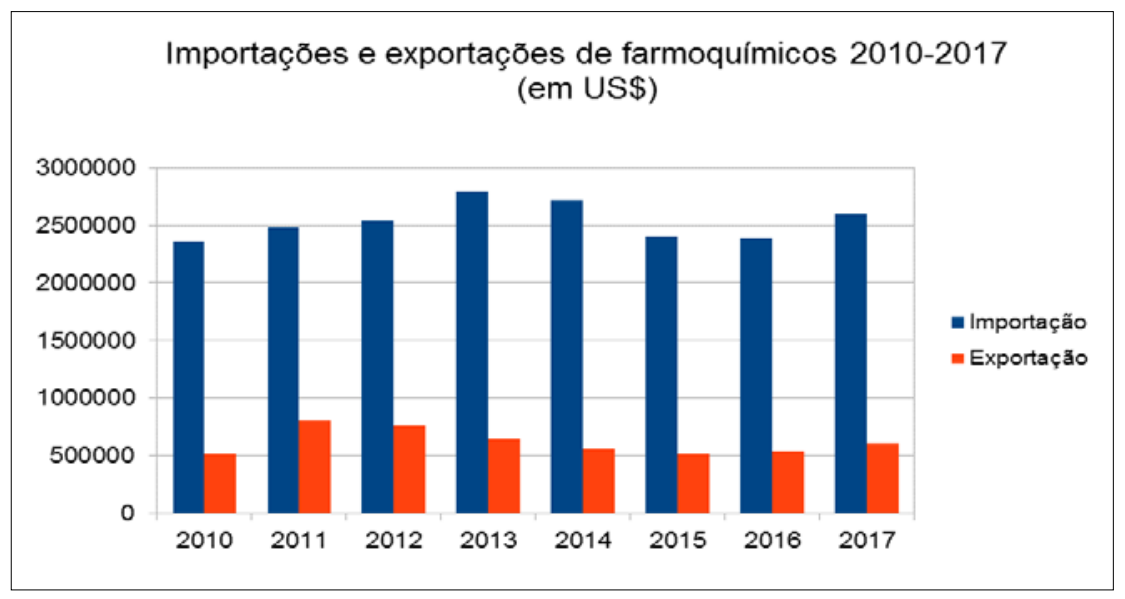

Gráfico 1 - Importações e exportações de farmoquímicos.

Fonte: Elaborado pelos autores com base nos dados extraídos da ABIFINA.

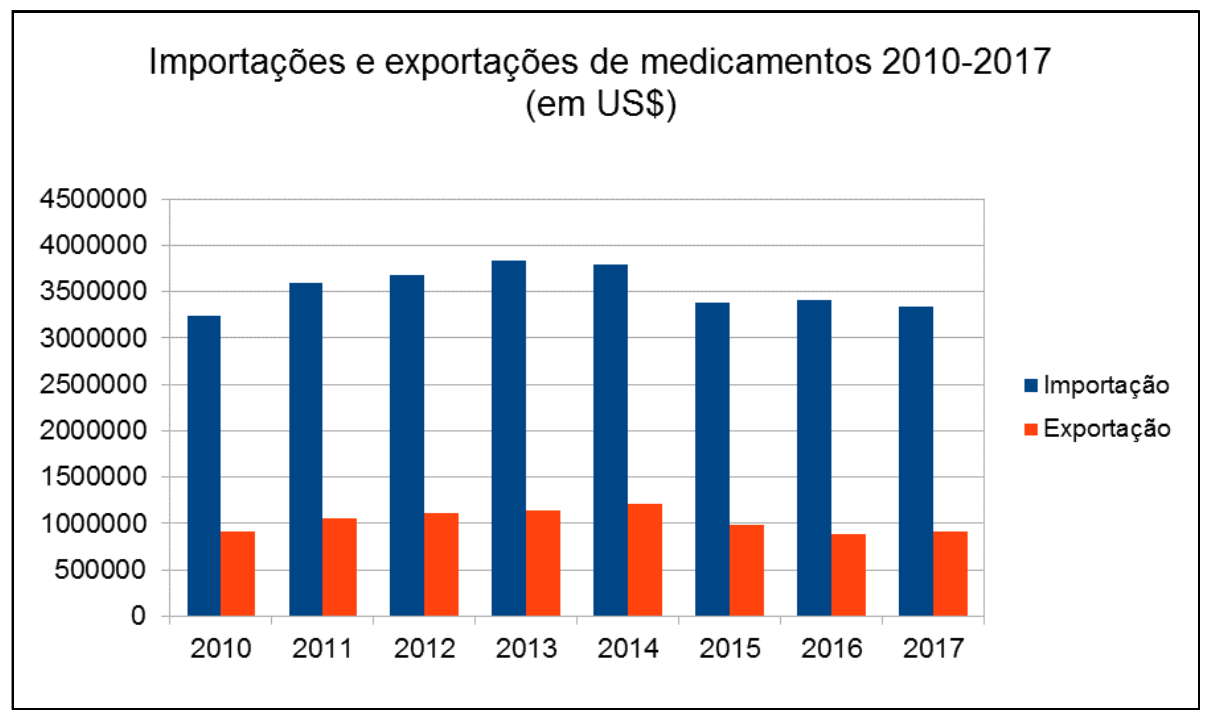

Gráfico 2 - Importações e exportações de medicamentos.

Fonte: Elaborado pelos autores com base nos dados extraídos da ABIFINA.

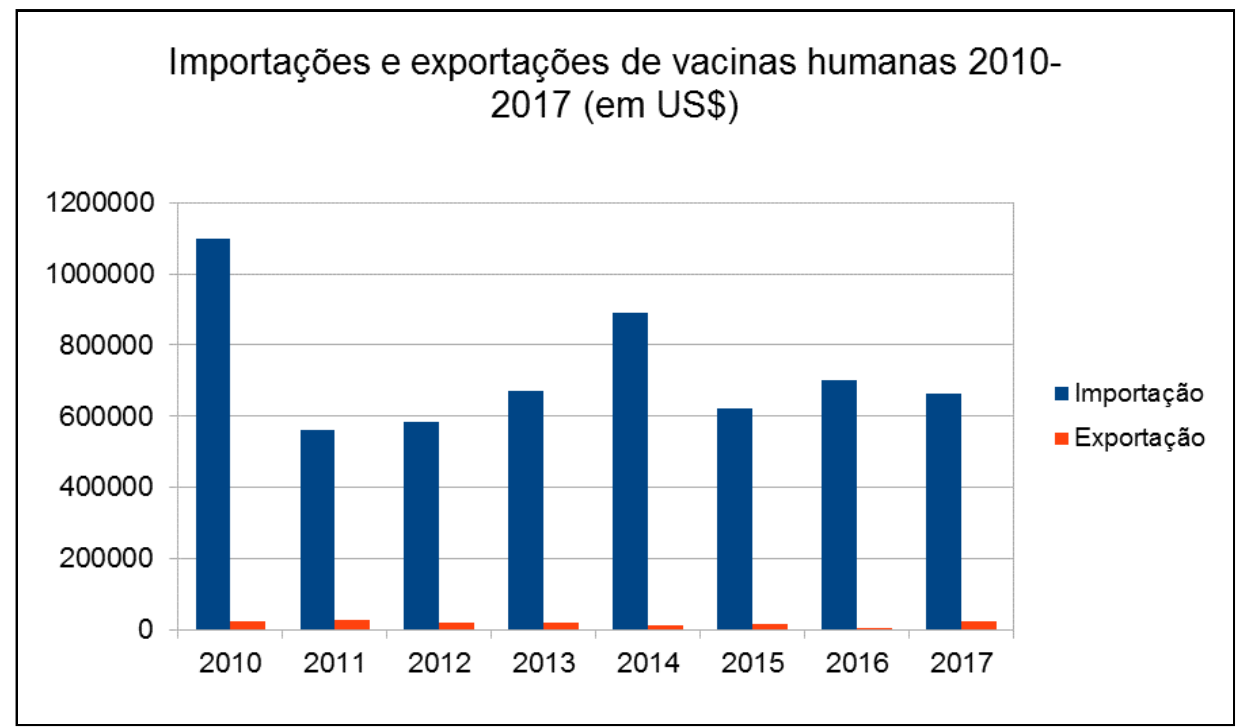

Gráfico 3 - Importações e exportações de vacinas humanas.

Fonte: Elaborado pelos autores com base nos dados extraídos da ABIFINA. 
Os dados mostram a enorme discrepância entre as importações e exportações de produtos essenciais do segmento de base química e biotecnológica, o que resulta em um enorme déficit comercial ano após ano, evidenciando a fragilidade da indústria brasileira e dependência do setor de saúde com relação ao mercado externo. A tabela 2 a seguir aponta quantitativamente o déficit apurado entre 2010 e 2017.

Tabela 2 - Déficit comercial das importações e exportações de medicamentos, farmoquímicos e vacinas humanas (2010-2017) - valores em US\$ MIL

\begin{tabular}{rccc}
\hline & Farmoquímicos & Medicamentos & Vacinas Humanas \\
\hline $\mathbf{2 0 1 0}$ & -1.847 .400 & -2.334 .353 & -1.074 .263 \\
\hline $\mathbf{2 0 1 1}$ & -1.676 .000 & -2.536 .109 & -533.300 \\
\hline $\mathbf{2 0 1 3}$ & -1.777 .900 & -2.566 .932 & -564.344 \\
\hline $\mathbf{2 0 1 4}$ & -2.148 .800 & -2.689 .419 & -653.644 \\
\hline $\mathbf{2 0 1 5}$ & -2.154 .900 & -2.581 .116 & -880.080 \\
\hline $\mathbf{2 0 1 6}$ & -1.894 .600 & -2.392 .097 & -605.157 \\
\hline $\mathbf{2 0 1 7}$ & -1.842 .600 & -2.525 .267 & -693.403 \\
\hline TOTAL & -1.983 .900 & -2.436 .746 & -639.109 \\
\hline
\end{tabular}

Fonte: Elaborada pelos autores com base nos dados extraídos da ABIFINA.

A tabela acima apenas corrobora os dados mostrados na tabela 1, porém, ao calcular o montante do déficit acumulado nos oito anos verificados, não há como não se alarmar e questionar o quanto as PDP têm, de fato, fortalecido o segmento de base química e biotecnológica no Brasil.

\subsection{Funcionamento das PDP}

Em face ao exposto anteriormente, surge a necessidade de explicar como funcionam as Parcerias para o Desenvolvimento Produtivo (PDP), conforme legislação atual sobre a matéria.

O art. $3^{\circ}$ do Anexo XCV da Portaria $n^{\circ}$ 5/2017, apresenta quais são os objetivos das PDP:

I - ampliar o acesso da população a produtos estratégicos e diminuir a vulnerabilidade do SUS;

II - reduzir as dependências produtiva e tecnológica para atender as necessidades de saúde da população brasileira a curto, médio e longo prazos, seguindo os princípios constitucionais do acesso universal e igualitário às ações e aos serviços de saúde;

III - racionalizar o poder de compra do Estado, mediante a centralização seletiva dos gastos na área da saúde, com vistas à sustentabilidade do SUS e à ampliação da produção no País de produtos estratégicos;

IV - proteger os interesses da Administração Pública e da sociedade ao buscar a economicidade e a vantajosidade, considerando-se preços, qualidade, tecnologia e benefícios sociais;

V - fomentar o desenvolvimento tecnológico e o intercâmbio de conhecimentos para a inovação no âmbito das instituições públicas e das entidades privadas, contribuindo para o desenvolvimento do CEIS e para torná-las competitivas e capacitadas; 
VI - promover o desenvolvimento e a fabricação em território nacional de produtos estratégicos para o SUS;

VII - buscar a sustentabilidade tecnológica e econômica do SUS a curto, médio e longo prazos, com promoção de condições estruturais para aumentar a capacidade produtiva e de inovação do País, contribuir para redução do déficit comercial do CEIS e garantir o acesso à saúde; e

VIII - estimular o desenvolvimento da rede de produção pública no País e do seu papel estratégico para o SUS (BRASIL, 2017).

De maneira a cumprir com os objetivos estabelecidos, são especificados dez grupos, cada qual contendo uma lista de produtos estratégicos para o SUS, que é definida pelo GECIS e atualizada anualmente. Três desses grupos referem-se especificamente aos produtos pertencentes ao segmento de base química e biotecnológica do CEIS: fármacos, medicamentos e vacinas.

A lista de 2018 ainda não foi divulgada, tendo sido realizada unicamente consulta pública para sua composição por meio do Informe Técnico n 09/2017 do Ministério da Saúde, sendo que na lista já sugerida constavam 76 medicamentos elegíveis para apresentação de propostas de $\mathrm{PDP}^{4}$. A Portaria $n^{\circ}$ 704, de 8 de março de 2017, que contém a lista referente a esse ano, trazia 56 medicamentos elegíveis ${ }^{5}$.

É a partir da divulgação da lista dos produtos estratégicos que ocorre a etapa de execução das PDP. Varrichio (2017) resume as quatro fases que compõem essa etapa:

Na primeira fase, uma instituição pública submete uma proposta de projeto de PDP em parceria com entidade privada ao MS. Na segunda fase, caso a proposta seja aprovada, há a assinatura de um contrato entre as entidades privadas e públicas com o registro do produto. Na terceira fase, há o primeiro fornecimento para o MS, definindo, assim, o início do processo de transferência de tecnologia. Na quarta e última fase, há a internalização da tecnologia pelo laboratório público segundo a portabilidade tecnológica (VARRICHIO, 2017).

Analisando os dados disponíveis pelo Ministério da Saúde das PDP segundo fases do processo, obtêm-se as seguintes informações (tabela 3):

Tabela 3 - Número de PDP por fase do processo

\begin{tabular}{|c|c|}
\hline \multicolumn{2}{|c|}{ Fase I: Propostas de projetos de PDP (atualizado em 19/04/2016) } \\
\hline Medicamentos & Produtos \\
\hline 8 & 0 \\
\hline \multicolumn{2}{|c|}{ Fase II: Projetos de PDP com termo de compromisso assinado (atualizado em } \\
\hline \multicolumn{2}{|c|}{ 10/05/2018) } \\
\hline Medicamentos & Produtos \\
\hline 35 & 6 \\
\hline $\begin{array}{c}\text { Fase III: início da fase de execução do desenvolvimento do produto, transferência e } \\
\text { absorção de tecnologia de forma efetiva e celebração do contrato de aquisição do }\end{array}$ \\
\hline
\end{tabular}

4 Informe técnico disponível em: http://portalarquivos2.saude.gov.br/images/pdf/2017/dezembro/18/IT--9.pdf. Acesso em: 01/06/2018.

5 Lista completa disponível em: http://bvsms.saude.gov.br/bvs/saudelegis/gm/2017/prt0704_10_03_2017.html. Acesso em: 01/06/2018.

6 Disponível em: <http://portalarquivos2.saude.gov.br/images/pdf/2016/abril/20/Fase-I-Propostas-de-Projeto-de-PDP--medicamentos-no-site-em-19.04.2016.pdf>. Acesso em: 02/06/2018.

7 Disponível em: <http://portalarquivos2.saude.gov.br/images/pdf/2018/maio/11/Fase-II-Projetos-de-PDP.pdf> e em $<$ http://portalarquivos2.saude.gov.br/images/pdf/2018/marco/20/Fase-II-Projetos-de-PDP.pdf>. Acesso em: 02/06/2018. 


\begin{tabular}{|c|c|}
\hline $\begin{array}{c}\text { produto estratégico entre o Ministério da Saúde e a instituição pública (atualizado em } \\
\mathbf{1 0 / 0 5 / 2 0 1 8})^{\mathbf{8}}\end{array}$ \\
\hline Medicamentos & Produtos \\
\hline 20 & 3 \\
\hline Fase IV: internalização de tecnologia (atualizado em 10/05/2018) \\
\hline Medicamentos & Produtos \\
\hline 11 & 0 \\
\hline
\end{tabular}

Fonte: Elaborada pelos autores com base nos dados do Ministério da Saúde.

\subsection{Demanda do SUS}

Para compreender a importância das PDP dentro das políticas industriais no setor de saúde, faz-se necessário um breve levantamento de dados relacionado ao gasto do SUS com medicamentos. Vieira (2018) aponta que, "no total, o gasto do SUS com medicamentos nas três esferas de governo passou, em termos reais, de R \$ 14,3 bilhões em 2010 para quase R\$ 20 bilhões em 2015 (crescimento de 40\%), caindo para R\$18,6 bilhões em 2016”.

O levantamento realizado pela autora englobou diversos programas e ações que sobrepassam o programa de assistência farmacêutica, abrangendo itens como implantação e manutenção das Farmácias Populares, imunobiológicos, segurança transfusional, entre outros que fogem ao escopo do presente trabalho.

Sendo assim, foram extraídos dados do Sistema de Informações sobre Orçamentos Públicos em Saúde (SIOPS), do período de 2013 a 2017, que mostram as despesas realizadas pela União por bloco de financiamento, sendo um deles o da assistência farmacêutica do SUS, cujos valores são apresentados no gráfico 4 a seguir, tendo sido considerados os valores informados com relação às despesas liquidadas, que são aquelas que já foram previamente empenhadas e cujo produto e nota fiscal foi entregue pelo fornecedor, sendo a dívida reconhecida pelo órgão:

Gráfico 4 - Despesas da União com assistência farmacêutica no período de 2013 a 2017

8 Disponível em: <http://portalarquivos2.saude.gov.br/images/pdf/2018/maio/11/Fase-III-Parcerias-para-oDesenvolvimento-Produtivo-PDP.pdf $>$ e $\quad<$ http://portalarquivos2.saude.gov.br/images/pdf/2018/marco/20/Fase-IIIPDP.pdf>. Acesso em: 02/06/2018.

9 Disponível em: <http://portalarquivos2.saude.gov.br/images/pdf/2018/maio/11/Fase-IV-Internalizacao-daTecnologia.pdf>. Acesso em: 02/06/2018. 
Fonte: Elaborado pelos autores com base nos dados extraídos do SIOPS.

Cabe destacar que esses gastos não se relacionam com as PDP e que a grande diferença de gastos verificada em 2017 se refere à redução no orçamento destinado a esse programa, que vinha se mantendo relativamente estável desde 2013, mas que nesse ano especificamente sofreu um corte de mais de $40 \%$ em comparação a 2016, passando de $\mathrm{R} \$ 4.666 .454 .657,63$ para $\mathrm{R} \$ 2.762 .148 .073,55$.

Depreende-se dos dados apresentados neste apartado, em comparação com os valores de importação de insumos do segmento de base química e biotecnológica no Brasil, que o SUS representa um (se não o maior) comprador de fármacos e medicamentos no país.

Com relação às compras via PDP, Varrichio (2017) aponta que "as aquisições de medicamentos e produtos realizadas pelo SUS, somente no âmbito das PDPs, entre 2009 e 2015, totalizaram R\$ 9,1 bilhões”. Em apresentação elaborada pelo Ministério da Saúde (2017), afirma-se que cerca de 30\% das compras do governo são por meio de PDP.

Dados disponibilizados pela Interfarma (Associação da Indústria Farmacêutica de Pesquisa, mostram que até março de 2016 haviam 76 parcerias vigentes, envolvendo 55 instituições privadas e 19 laboratórios públicos, com um resultado de 33 produtos registrados na Agência Nacional de Vigilância Sanitária (ANVISA) e uma economia de R\$ 1,6 bilhão entre 2011 e 2014 (Interfarma, 2016).

Em 2017 foram contabilizadas 74 PDP vigentes dentro das quatro fases de execução estabelecidas pela Portaria que regulamenta as parcerias, sendo 44 medicamentos e cinco vacinas, com uma estimativa de economia de R 5,2 bilhões desde 2010.

Não foram localizados dados sobre gastos por meio de PDP específicos com medicamentos. No entanto, com a intenção de buscar mostrar os gastos realizados com aquisição de produtos do segmento da base química e biotecnológica por meio de PDP, foi analisado um documento disponibilizado pelo Ministério da Saúde, cujo conteúdo recebeu sua última atualização em 19/05/2017. Esse registro apresenta uma lista de todas as aquisições realizadas, separada por ano, nome do medicamento, laboratório responsável, modalidade de compra e o link do diário oficial contendo a publicação referente à aquisição ${ }^{10}$. Sendo assim, foi verificado cada um dos 105 links disponíveis para identificar o valor total das aquisições. Os resultados apurados são mostrados abaixo separados apenas pelo valor gasto por exercício financeiro, sem especificação de cada medicamento (tabela 5). Considerando que os dados foram atualizados no primeiro semestre de 2017, serão apresentados os valores de 2010 a 2016.

Tabela 5 - Gastos com aquisição de produtos do segmento de base química e biotecnológica por meio de PDP (2010 a 2016)

\begin{tabular}{cc}
\hline ANO & VALOR \\
\hline 2010 & $\mathrm{R} \$ 105.300 .000,00$ \\
\hline 2011 & $\mathrm{R} \$ 667.840 .908,64$ \\
\hline 2012 & $\mathrm{R} \$ 1.066 .232 .537,88$ \\
\hline 2013 & $\mathrm{R} \$ 1.694 .291 .569,95$ \\
\hline 2014 & $\mathrm{R} \$ 2.646 .683 .040,05$ \\
\hline 2015 & $\mathrm{R} \$ 3.404 .339 .526,42$ \\
\hline 2016 & $\mathrm{R} \$ 2.982 .107 .536,82$ \\
\hline TOTAL & $\mathbf{R} \mathbf{1 2 . 5 6 6 . 7 9 5 . 1 1 9 , 7 6}$ \\
\hline
\end{tabular}

Fonte: Elaborada pelos autores.

10 Disponível em: <http://portalarquivos2.saude.gov.br/images/pdf/2017/maio/22/Aquisicoes\%20de\%20produtos\%20de\%20PDP \%20atualizada\%20em\%2019.05.2017.pdf>. Acesso em: 02/06/2018. 
Há de se reconhecer que os gastos com produtos do segmento de base química e biotecnológica mostram um crescimento anual das PDP, evidenciando um fortalecimento das políticas industriais do setor de saúde. É um cenário ainda longe do ideal em termos de desenvolvimento nacional, em face aos números apresentados em relação às importações e exportações, mas já é possível vislumbrar um crescimento promissor, desde que as políticas industriais e as ações de PDP sejam perseguidas como agenda nacional, e não simples aparato governamental com prazo de validade.

\section{AS PDP E O FORTALECIMENTO DO SEGMENTO DE BASE QUÍMICA E BIOTECNOLÓGICA NO BRASIL}

Os dados apresentados no decorrer deste trabalho permitem inferir a importância das políticas industriais na área da saúde e a necessidade de uma visão schumpeteriana da busca pela inovação e de uma agenda que priorize o desenvolvimentismo nacional.

As Parcerias para o Desenvolvimento Produtivo são ações que seguem essa linha ao incentivar cooperações entre instituições no intuito de aprimorar a produção nacional por meio do desenvolvimento, transferência e absorção de tecnologia, buscando tornar o país, e especificamente a área da saúde, menos vulnerável aos caprichos do mercado econômico externo.

Considerando que a saúde no Brasil é um direito garantido constitucionalmente, criou-se o Sistema Único de Saúde (SUS), com o dever de garantir a saúde por meio da formulação e execução de políticas econômicas e sociais que visem à redução de riscos de doenças e de outros agravos, além do estabelecimento de condições que assegurem acesso universal e igualitário às ações e aos serviços para a sua promoção, proteção e recuperação (BRASIL, 1990).

Nesse sentido, compreende-se que, para o cumprimento das disposições legais acerca do acesso à saúde, o SUS conta com produtos e serviços estratégicos para o atendimento das demandas populacionais. Esses produtos compõem o denominado Complexo Econômico-Industrial da Saúde, que engloba três segmentos principais: o de produtos para a saúde, o de serviços e o de base química e biotecnológica.

Com relação ao segmento de base química e biotecnológica, que abarca a indústria farmacêutica, as vacinas, os hemoderivados e reagentes para diagnósticos, dados apontam a fragilidade do setor industrial brasileiro na produção desse tipo de produtos, o que acarreta em custos elevados para o SUS e torna o país dependente da importação e suscetível às variações econômicas desse tipo de processo comercial.

As políticas industriais destacadas na segunda seção deste trabalho já vinham demonstrando uma crescente preocupação em aliar o processo desenvolvimentista ao setor de saúde, como forma de promoção do desenvolvimento econômico e social. A regulamentação das PDP em meio às ações de política industrial inseridas no Plano Brasil Maior garantiu um impulso ao desafio da capacitação e aprendizado tecnológico nacional ao assegurar incentivos à produção local, o que acarreta, também, na diminuição das fragilidades do SUS e em economia de recursos, que podem ser destinados a outras necessidades.

Os números apresentados revelam que uma gestão responsável, planejamento contínuo e acompanhamento das PDP resultam sim em um fortalecimento do segmento de base química e biotecnológica nacional.

Buscando realizar uma comparação simples entre os valores gastos com assistência farmacêutica fora do âmbito das PDP e as compras de medicamentos via parcerias (conforme dados apresentados no gráfico 4 e na tabela 5, delimitando o período entre 2013 e 2016), elaborou-se o gráfico 5, apresentado abaixo, que denota uma diferença cada vez menor entre os tipos de aquisições, mostrando que a continuidade das ações dentro dos objetivos estipulados pode fortalecer cada vez mais a indústria brasileira. Cabe enfatizar que essa comparação se baseia apenas nos valores financeiros, sem levar em consideração o tipo de medicamento adquirido, o que pode ser objeto futuro de uma análise específica. 


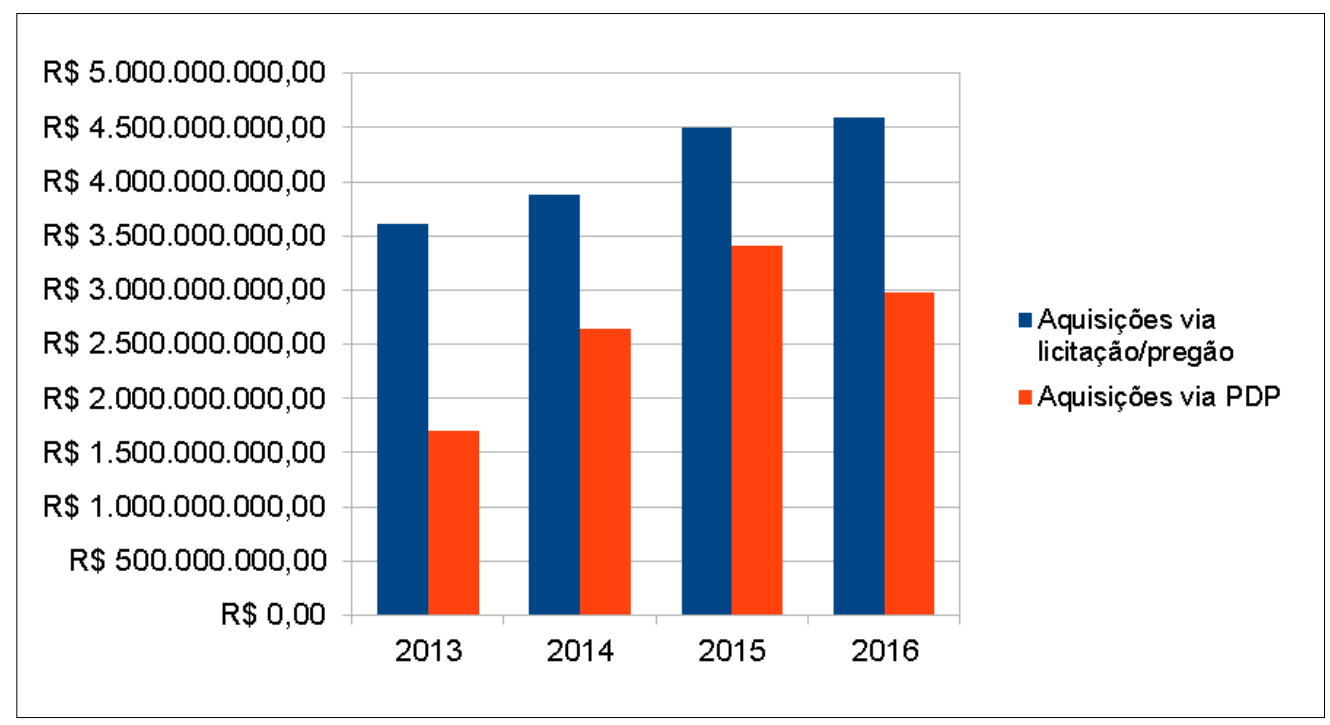

\section{Gráfico 5 - Comparativo de gastos entre as modalidades de aquisição de medicamentos} (2013-2016)

Fonte: Elaborado pelos autores

Portanto, reconhece-se dentro das PDP uma ferramenta favorável ao desenvolvimentismo nacional, cujas ações tendem a aprimorar a capacidade produtiva do país por meio do desenvolvimento e aprendizado tecnológico, resultando em melhoria ao atendimento das necessidades do setor de saúde e, consequentemente, do desenvolvimento econômico e social.

\section{CONCLUSÃO}

Em face a tudo o exposto no decorrer deste trabalho, é inegável o papel fundamental da saúde como um dos propulsores do desenvolvimento e crescimento nacional. Um país saudável é um país produtivo e cabe ao Estado garantir o acesso aos meios necessários para promoção, proteção e recuperação da saúde.

Dentro desse escopo, insere-se a obrigação de assegurar o fornecimento de produtos do segmento da base química e biotecnológica, em especial medicamentos e vacinas, além do aprimoramento do setor industrial em prol da pesquisa e produção desses insumos, de forma a diminuir a susceptibilidade do SUS aos caprichos econômicos do mercado internacional.

As Parcerias para o Desenvolvimento Produtivo surgem como uma ferramenta dentro das políticas industriais destinadas ao setor da saúde, especialmente após o lançamento do Plano Brasil Maior. Com o objetivo de fortalecer o CEIS, as PDP mostram-se essenciais para alicerçar a base industrial nacional, que após anos de abandono se encontrava estagnada, voltada à já ultrapassada política de substituição de importações e a uma produção essencialmente de commodities.

Assim, verifica-se nas PDP uma estratégia, inclusive, de negociação, pois é inegável que o SUS é um dos (se não o maior) compradores de medicamentos no país. Sendo assim, o poder público detém em suas mãos um poder de permuta perante o mercado internacional, que pode atrair a transferência tecnológica e impulsionar o desenvolvimento tecnológico nacional.

Conclui-se, portanto, que as PDP devem continuar sendo utilizadas dentro das políticas industriais de saúde com o objetivo primordial de inovar e promover ações que condicionem e favoreça o desenvolvimentismo capitalista nacional, de forma a auxiliar o alcance de um desenvolvimento econômico e social capaz de superar as barreiras da desigualdade e buscar garantir um crescimento contínuo e sustentável. 


\section{REFERÊNCIAS}

ABDI. Política Industrial, Tecnológica e de Comércio Exterior - PITCE Balanço e Perspectivas. Agência Brasileira de Desenvolvimento Industrial - ABDI. OUTUBRO/2006. Disponível em: $<$ http://www.abdi.com.br/Estudo/Balanco\%20PITCE\%20nov2006.pdf>. Acesso em: 23/05/2018.

ABIFINA. Estatísticas. Disponível em: <http://www.abifina.org.br/estatisticas_saldo.php>. Acesso em: 23/05/2018.

BIELSCHOWSKY, Ricardo. Sesenta años de la cepal: estructuralismo y neoestructuralismo. Revista CEPAL 97, Abril 2009.

BRASIL. Ministério da Saúde. Portaria $\mathrm{n}^{0}$ 374, de 28 de fevereiro de 2008. Institui no âmbito do Sistema Único de Saúde - SUS, o Programa Nacional de Fomento à Produção Pública e Inovação no Complexo Industrial da Saúde. Brasília, 2008a. Disponível em: <http://bvsms.saude.gov.br/bvs/saudelegis/gm/2008/prt0374_28_02_2008.html>. Acesso em: 24/05/2018.

BRASIL. Decreto de 12 de maio de 2008. Cria, no âmbito do Ministério da Saúde, o Grupo Executivo do Complexo Industrial da Saúde- GECIS, e dá outras providências. Revogado pelo Decreto $n^{\circ}$ 9.245, de 2017. Disponível em: <http://www.planalto.gov.br/ccivil_03/_Ato2007-2010/2008/Dnn/Dnn11578.htm>. Acesso em: 24/05/2018.

BRASIL. Decreto $n^{0}$ 7.540, de 02 de agosto de 2011. Institui o Plano Brasil Maior - PBM e cria o seu Sistema de Gestão. Brasília, 2011. Disponível em: <https://www.planalto.gov.br/ccivil_03/_ato20112014/2011/decreto/d7540.htm>. Acesso em: 24/05/2018.

BRASIL. Plano Brasil Maior: Inovar para competir. Competir para crescer. Disponível em: <file:///D:/Artigo\%20Malu/plano_brasil_maior.pdf>. Acesso em: 26/05/2018.

BRASIL. Ministério da Saúde. Gabinete do Ministro. Portaria ${ }^{\circ}$ 837, de 18 de abril de 2012: define as diretrizes e os critérios para o estabelecimento das parcerias para o desenvolvimento produtivo (PDP). Disponível em: <http://bvsms.saude.gov.br/bvs/saudelegis/gm/2012/prt0837_18_04_2012.html>. Acesso em: 28/05/2018.

BRASIL. Constituição da República Federativa do Brasil. Brasília: Supremo Tribunal Federal, Secretaria de Documentação, 1988.

BRASIL. Lei $n^{\circ}$ 8.080, de 19 de setembro de 1990. Dispõe sobre as condições para promoção, proteção e recuperação da saúde, a organização e o funcionamento dos serviços correspondentes e dá outras providências. Brasília, 1990. Disponível em: <http://www.planalto.gov.br/ccivil_03/leis/L8080.htm>. Acesso em: 01/06/2018.

BRASIL. Ministério da Saúde. Secretaria de Ciência, Tecnologia e Insumos Estratégicos. Departamento de Ciência e Tecnologia. Política nacional de ciência, tecnologia e inovação em saúde / Ministério da Saúde, Secretaria de Ciência, Tecnologia e Insumos Estratégicos, Departamento de Ciência e Tecnologia - 2. ed.- Brasília : Editora do Ministério da Saúde, 2008b.

BRASIL. Ministério da Saúde. Portaria $n^{0}$ 506, de 21 de março de 2012. Institui o Programa para o Desenvolvimento do Complexo Industrial da Saúde (PROCIS) e seu Comitê Gestor. 2012. Disponível em: <http://bvsms.saude.gov.br/bvs/saudelegis/gm/2012/prt0506_21_03_2012.html>. Acesso em: 01/06/2018. 
BRASIL. Ministério da Saúde. Secretaria-Executiva. Subsecretaria de Planejamento e Orçamento. Plano Nacional de Saúde - PNS: 2012-2015 / Ministério da Saúde. Secretaria-Executiva. Subsecretaria de Planejamento e Orçamento. - Brasília: Ministério da Saúde, 2011.

BRASIL. Ministério da Saúde. Secretaria de Políticas de Saúde. Departamento de Atenção Básica. Política nacional de medicamentos 2001/Ministério da Saúde, Secretaria de Políticas de Saúde, Departamento de Atenção Básica. - Brasília : Ministério da Saúde, 2001.

BRASIL. Ministério da Saúde. Despesa por bloco de financiamento. SIOPS/DATASUS. Disponível em: $<$ http://siops.datasus.gov.br/relUN.php?acao=3>. Acesso em 01/06/2018.

BRASIL. Ministério da Saúde. Portaria $n^{0}$ 704, de 8 de março de 2017. Define a lista de produtos estratégicos para o Sistema Único de Saúde (SUS), nos termos dos anexos a esta Portaria. Disponível em: <http://bvsms.saude.gov.br/bvs/saudelegis/gm/2017/prt0704_10_03_2017.html>. Acesso em: 01/06/2018.

BRASIL. Ministério da Saúde. Audiência Pública - a necessidade de Regulamentação da Intercambialidade entre o produto originador e biossimilar. Brasília, 2017. Disponível em: $<$ http://www2.camara.leg.br/atividade-legislativa/comissoes/comissoes-permanentes/cctci/audienciaspublicas/2017/2017-11-23-apc-a-intercambialidade-entre-o-produto-originador-e-o-biossimilar/ricardobarcelos-ms>. Acesso em: 01/06/2018.

BRASIL. Ministério da Saúde. Portaria de Consolidação GM/MS nº 5/2017. Consolidação das normas sobre as ações e os serviços de saúde do Sistema Único de Saúde. Anexo XCV. Dos critérios para a definição da lista de produtos estratégicos para o SUS e do estabelecimento das parcerias para o desenvolvimento produtivo (PDP) e dos respectivos processos de submissão, instrução, decisão, transferência e absorção de tecnologia, aquisição de produtos estratégicos para o SUS no âmbito das PDP e o respectivo monitoramento e avaliação. Brasília, 2017. Disponível em: $<$ http://bvsms.saude.gov.br/bvs/saudelegis/gm/2017/prc0005_03_10_2017.html>. Acesso em: 02/06/2018.

BRESSER-PEREIRA, Luiz Carlos; GALA, Paulo. Macroeconomia estruturalista do desenvolvimento. Revista de Economia Política, vol. 30, nº 4 (120), pp. 663-686, outubro-dezembro/2010.

CARRARA, Bruna Sordi; VENTURA, Carla Aparecida Arena. A saúde e o desenvolvimento humano. Sau. \&Transf. Soc., ISSN 2178-7085, Florianópolis, v.3, n.4, p.89-96, 2012.

CASTRO, Lavinia Barros de. Esperança, Frustração e Aprendizado: A História da Nova República (19851989). In Economia brasileira contemporânea [recurso eletrônico]: 19452010 / [organizadores Fabio Giambiagi... et al.]. - Rio de Janeiro: Elsevier, 2011.

COSTA, Lais Silveira; METTEN, Antoine; DELGADO, Ignácio José Godinho. As Parcerias para o Desenvolvimento Produtivo em saúde na nova agenda de desenvolvimento nacional. Saúde e Debate. Rio de Janeiro, v. 40, n. 111, p. 279-291, out-dez 2016.

GADELHA, Carlos Augusto Grabois. Saúde e desenvolvimento: uma nova abordagem para uma nova política. Rev Saúde Pública, 46(Supl):5-8, 2012.

GADELHA, Carlos Augusto Grabois. Desenvolvimento e Saúde: em busca de uma nova utopia. Saúde em Debate; Rio de Janeiro, v. 19, n. 71, p. 326-327, set/dez. 2007. 
Gadelha, CAG; Barbosa, PR; Maldonado, J.; Vargas, M.; Costa, L. O Complexo Econômico-Industrial da Saúde: conceitos e características gerais. In Informe CEIS. VPPIS/FIOCRUZ - Informe CEIS n ${ }^{\circ}$ 1, ano 1 - Agosto 2010.

GADELHA, Carlos Augusto Grabois. O complexo industrial da saúde e a necessidade de um enfoque dinâmico na economia da saúde. Revista Ciência e Saúde Coletiva, 8(2): 521-535, 2003.

G1. BNDES renova o profarma até 2012, com orçamento de R\$ 3 bilhões. Disponível em: <http://g1.globo.com/Noticias/Economia_Negocios/0,,AA1656877-9356,00-

BNDES+RENOVA+O+PROFARMA+ATE+COM+ORCAMENTO+DE+R+BILHOES.html>. Outubro, 2017. Acesso em: 22/05/2018.

HASENCLEVER, Lia. Desafios de operação e desenvolvimento do complexo industrial da saúde [recursao eletrônico] / organização Lia Hasenclever et al. 1ª ed. Rio de Janeiro: E-papers, 2016.

INTERFARMA. Parcerias para o Desenvolvimento Produtivo (PDP). Disponível em: $<$ https://www.interfarma.org.br/guia/guia_2016/parcerias_para_desenvolvimento_produtivo/>. Acesso em: 01/06/2018.

KOHLI, Atul. Coping with globalization: Asian versus Latin American strategies of development, 19802010. Brazilian Journal of Political Economy. Vol. 32, nº 4 (129). pp 531-556. October-December/2012.

KUPFER, David; HASENCLEVER, Lia. Economia industrial: fundamentos teóricos e práticas no Brasil. Rio de Janeiro, RJ: Campus; Elsevier, 2002.

PEREZ, Carlota. Technological revolutions and techno-economic paradigms. TOC/TUT Working Paper No. 20 Working Papers in Technology Governance and Economic Dynamics The Other Canon Foundation, Norway and Tallinn University of Technology, January 20, 2009.

SUZIGAN, Wilson; FURTADO, João. Política Industrial e Desenvolvimento. Revista de Economia Política, vol. 26, nº 2 (102), pp. 163-185 abril-junho/2006.

TORRES, Ricardo Lobato. A política de desenvolvimento da indústria farmacêutica brasileira de 2003 a

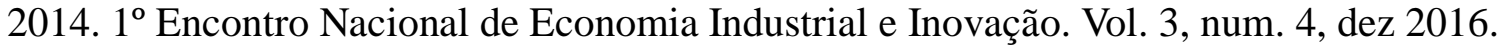

VARRICHIO, Pollyana C. SUS: o maior comprador de medicamentos do país. Radar, 52, ago. 2017. Disponível em: <http://repositorio.ipea.gov.br/bitstream/11058/8021/1/Radar_n52_SUS.pdf>. Acesso em: 01/06/2018.

VIEIRA, Fabiola Sulpino. Evolução do gasto com medicamentos do Sistema Único de Saúde no período de 2010 a 2016. Texto para discussão. Instituto de Pesquisa Econômica Aplicada.- Brasília : Rio de Janeiro: $\quad$ Ipea, $2018 . \quad$ Disponível em: <http://www.ipea.gov.br/portal/images/stories/PDFs/Tds/180117_td_2356.pdf>. Acesso em: 01/06/2018.

WHO [OMS]. Macroeconomics and health: Investing in health for economic development. Report of the Commission on Macroeconomics and Health, 2001. 